\title{
An Ecological Survey of Benthic Invertebrates in Three Sites in Shatt-Al-Kufa at Al-Najaf Province, Iraq
}

\author{
Feryal A. M. Al-Ameen \\ Department of Biology, College of Science, University of Kufa.Kufa, Iraq.
}

feryalameen11@gmail.com

Keywords: Shatt Al-kufa, water, benthic invertebrate.

\begin{abstract}
The present study was conducted to determine the structure of benthic invertebrates community, as well as study of some factors associated with water quality in Shatt Al-kufa. The study was included a choice of three sites located along the Shatt Al-Kufa River, water samples and benthic invertebrates were collected during the period from February 2014 to January 2015.

The abiotic study included measurements of chlorophyll a, salinity, total dissolved solids, biochemical oxygen demand, total hardness, nitrate and sulphate. The biotic study included the determination the composition of the benthic invertebrates community through the study of the mean population density, the relative abundance index of these organisms and the Jaccard Coefficient was calculated to identify the value of similarity between the studied sites. In the present study 28 taxa of benthic invertebrates were recorded belong to 4 main groups which are: 8 taxa belonged to Annelida, 7 belonged to Insecta, 10 belonged to Mollusca, 3 belonged to Nematoda. Annelida recorded the highest percentage $40.8 \%$ of the total number of benthic invertebrates, Insecta with $30.3 \%$, Mollusca and Nematoda with $28.4 \%, 0.5 \%$ respectively. Benthic invertebrate have shown positive and negative relationships with the studied physical and chemical characteristics.
\end{abstract}

\section{مسح بيئي للافقريات القاع في ثلاث مواقع في شط الكوفة في محافظة النجف, العراق \\ فريال امين مرزة الامين

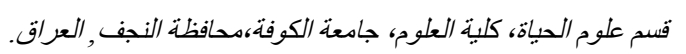

feryalameen11@gmail.com 
\% ثم النو اعم والديدان الخيطية بنسبة ع. r \% \%, 0. .\% على التو الي. وقد اظهرت اللافقريات القاعية علاقات موجبة وسالبة مع العوامل الفيزيائية و الكيميائية المدروسة. الكلمات الدالة: شط الكوفة, المياه, الديدان الحلقية.

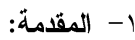

ان مجتمع اللافقريات المائية يضم مجموعة من الأحياء منها مايدعى باللافقريات القاعية التي وصفها كلا من [1] [2] على إنها حيو انات فاقدة

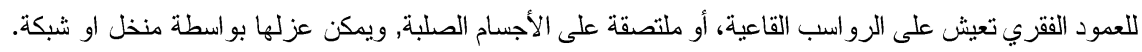

تمتلك لافقريات القاع اهمية كبيرة من الناحية الاقتصادية والعلمية, فهي تعد مادة غذائية غنية بالبروتين للاحياء المائية الاخرى, فضدلا عن انها

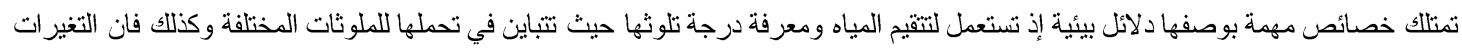

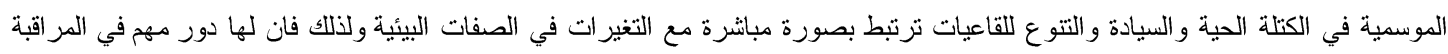

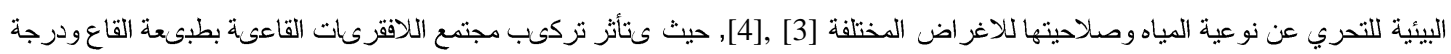

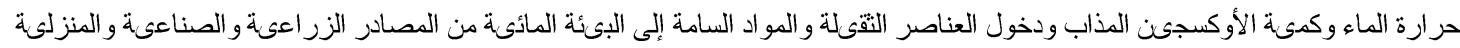

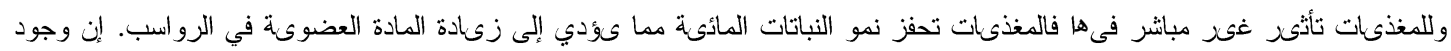

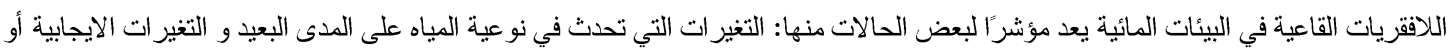

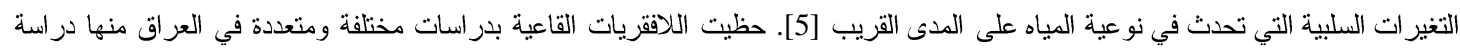

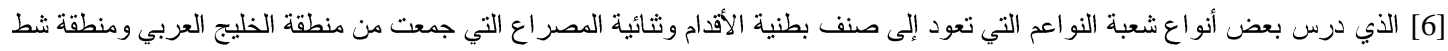

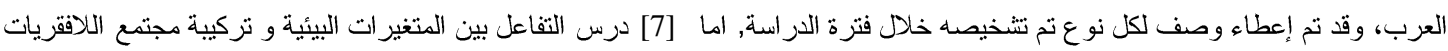

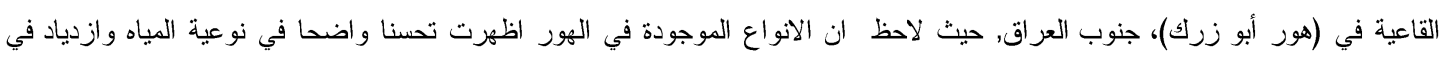

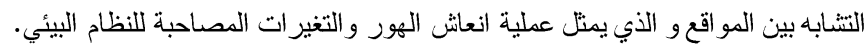

هدفت الدراسة الحالية الى تحديد تركيب مجتمع اللافقريات القاعية في ثلاثة مو اقع على شط الكوفة ومعرفة الوفرة النسبية لهذه الكائنات.

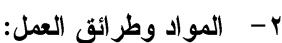

يعد نهر الفر ات من الانهار المهمة وعند دخوله الار اضي العراقية يشكل منطقة دلتا عند مدينة هيت والرمادي، وفي مدينة الكفل يتفرع نهر

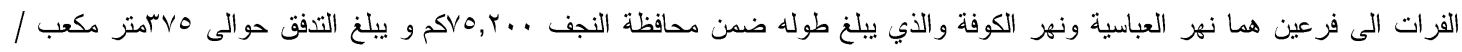

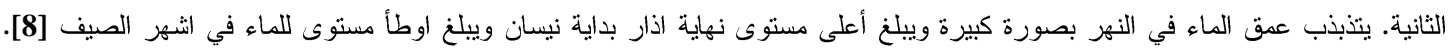

$$
\text { لتحقيق اهداف الدراسة تم اختيار ثثلاث مواقع على نهر الكوفة وكما يلي (ثكل ()): }
$$

ا-الموقع الاول (St.1): ويقع بالقرب من دار ضيافة المحافظة و يصب في هذا الموقع مجزى لصرف مياه الامطار وهي جزء من الثبكة القديمة لمدينة الكوفة.

r- الموقع الثاني (2. 2) : ويقع بعد التقاء الفرع الجانبي لنهز الكوفة ويمتد لمسافة ب كم من الموقع الاول. r- الموقع الثالث (3t. St): ويقع بالقرب من جسر البو عيسى ويظهر في هذا الموقع تاثثر مياه محطة لمعالجة المياه النقيلة لمدينتي النجف و الكوفة.

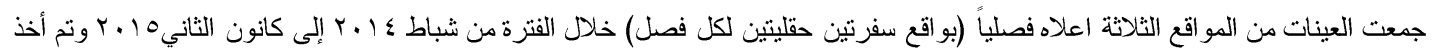

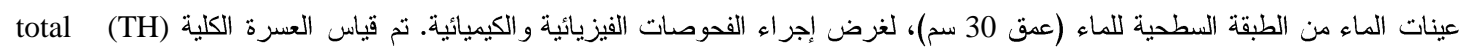

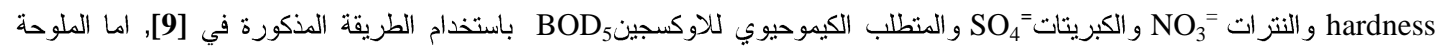
salinity والمواد الصلبة الذائبة الكلية وليست بصورة مباثرة في الحقل بإستخدام جهاز متعدد القياسات الرقمي المحمول بعد معايرته

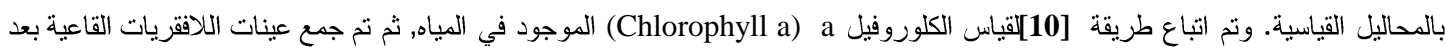

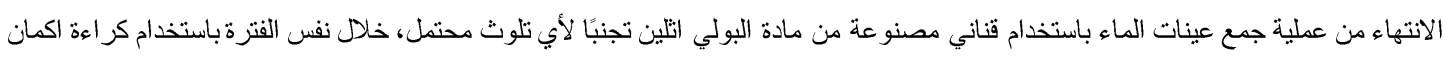

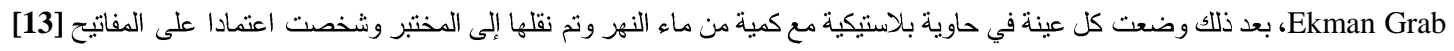

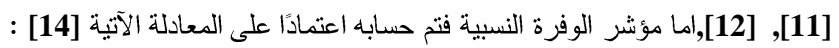


مؤشر الوفزة النسبية(Ra) Relative Abundance ( Ra Ns

إذ إن :

N : تمثل عدد الأفر اد العائدين لكل وحدة تصنيفية. :تمثل العدد الكلي للأفر اد في العينة. Ns

و قد حددت النتائج باستخدام النسبة المئوية وكما يلي-: الوحدات التصنيفية السائدة Dominant Taxa 70 \% الوحدات التصنيفية الوفيرة Abundant Taxa بين(40 - 70 \%). الوحدات التصنيفية الاقل وفرة Less Abundant Taxa بين( 10- 40 \%). الوحدات التصنيفية النادرة Rare Taxa 10 \%

اما معامل جاكارد للنتابه فتم حسابة وفقا للمعادلة التي وردت في [15]:-

$$
(C j=j /(a+b-j)
$$

عدد الوحدات التصنيفية المشتركة في كلتا العينتين. :J

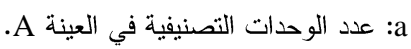

B عدد الوحدات التصنيفية في العينة الوحنة التصنة : b

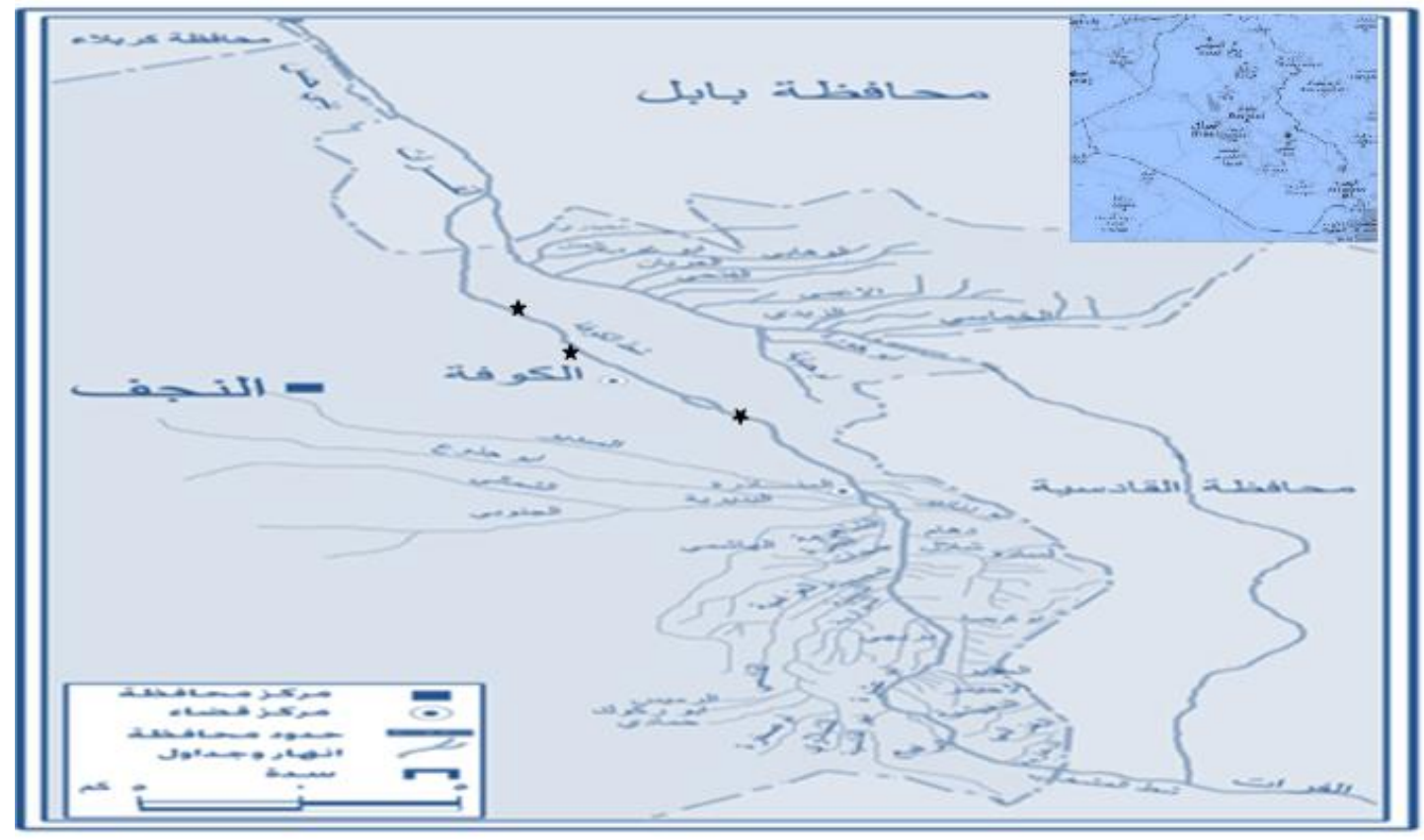

شكل(1): يبين مواقع الار اسة على شط الكوفة 


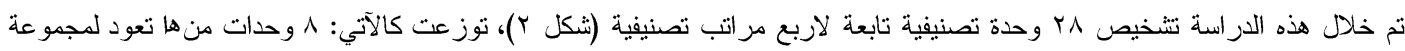

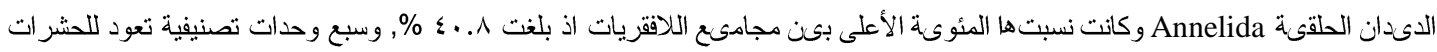

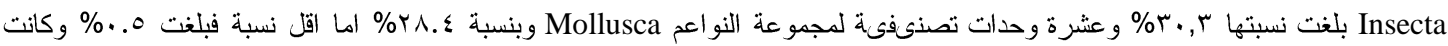

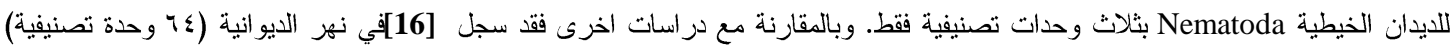

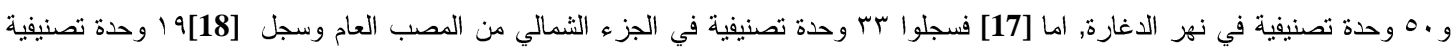

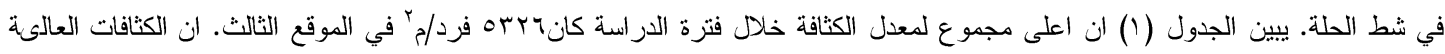

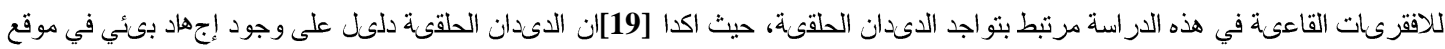

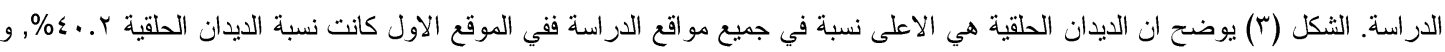

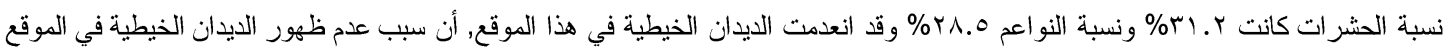

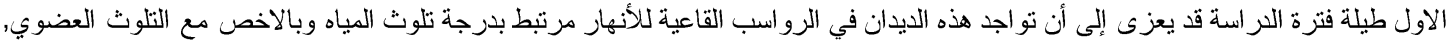

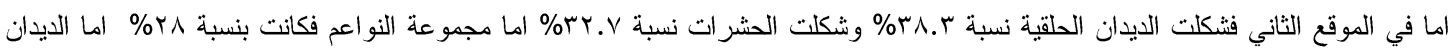

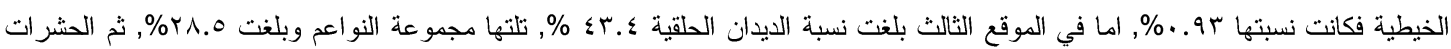

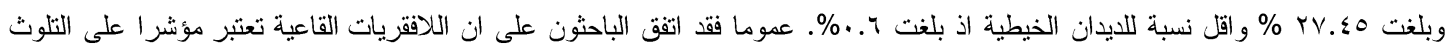

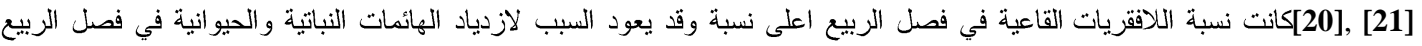

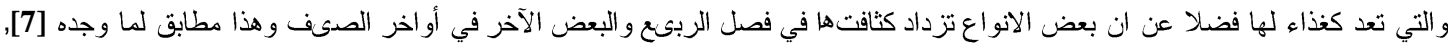

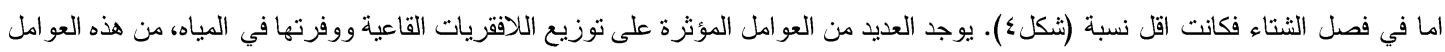

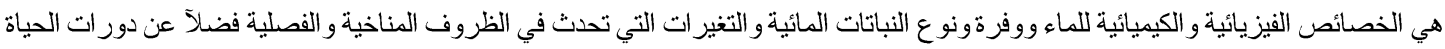

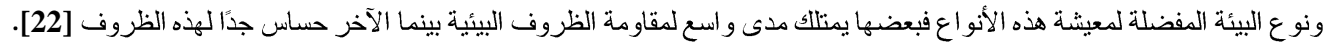

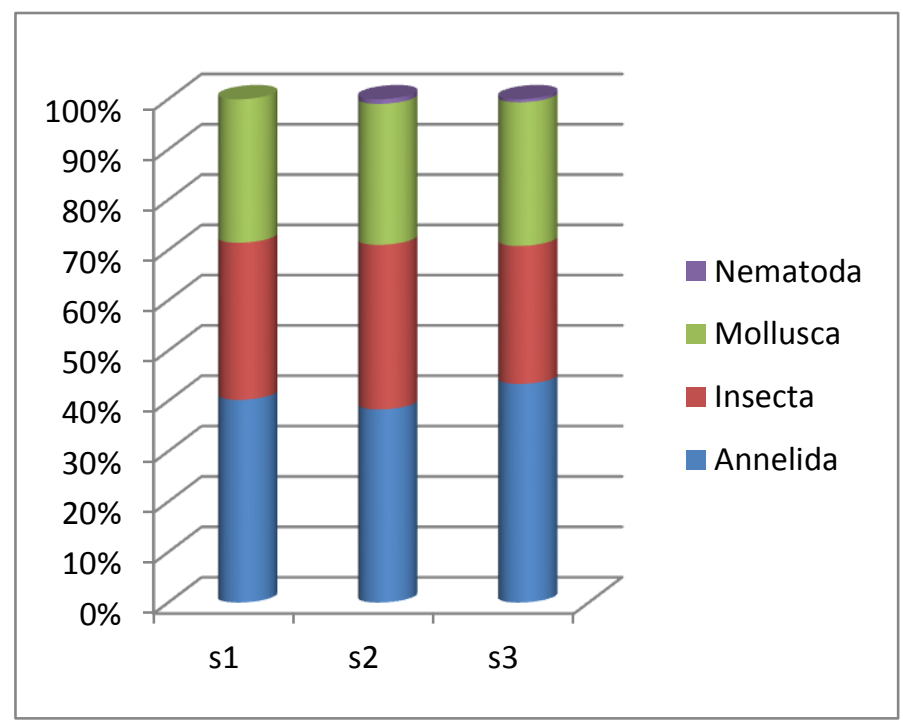

شكل(ץ): يبين النسب المئوية لمجاميع اللافقريات القاعية. 
جدول(1):معلد الكثافة (فرد/م") خلا فترة الدراسة.

\begin{tabular}{|c|c|c|c|}
\hline S3 & S2 & S1 & المجاميع اللافقرية \\
\hline YTIT & 1101 & 1941 & Annelida \\
\hline TETY & $10 \times 9$ & $1 \leqslant 99$ & Insecta \\
\hline 10rY & Irov & 1470 & Mollusca \\
\hline$r$. & $\leqslant 0$ & . & Nematoda \\
\hline OrYT & EATK & $\varepsilon \vee V \lambda$ & المجموع \\
\hline
\end{tabular}

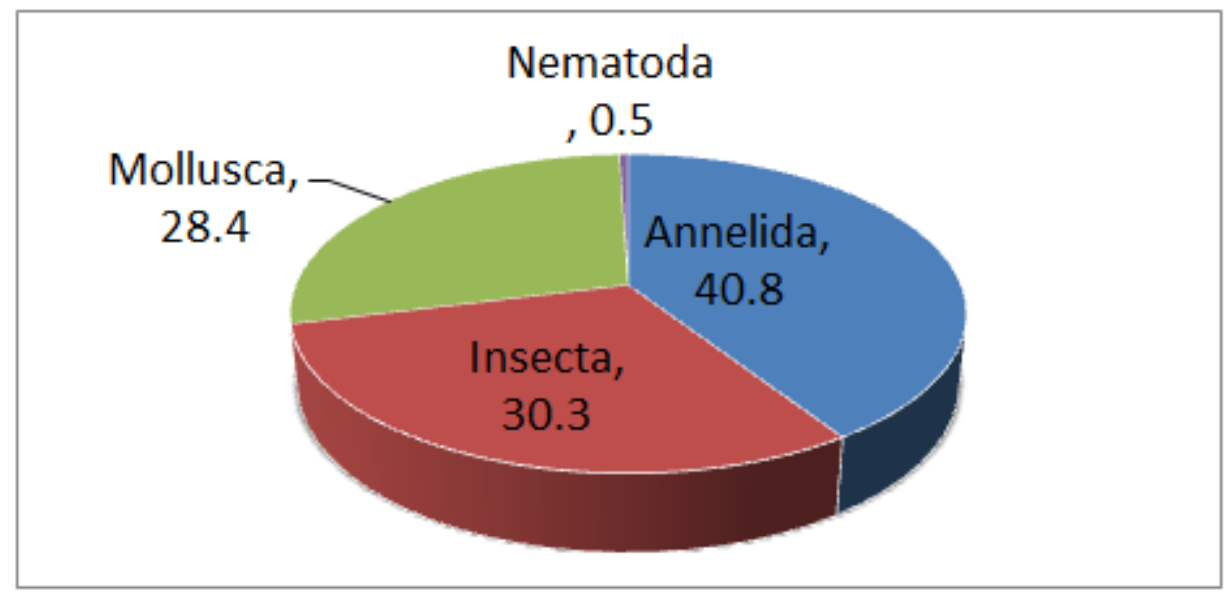

شكل(r): النسب المئويِة للوحدات التصنيفيةة الرئيسة في مواقع الار اسنة خلا فترة الاراسة

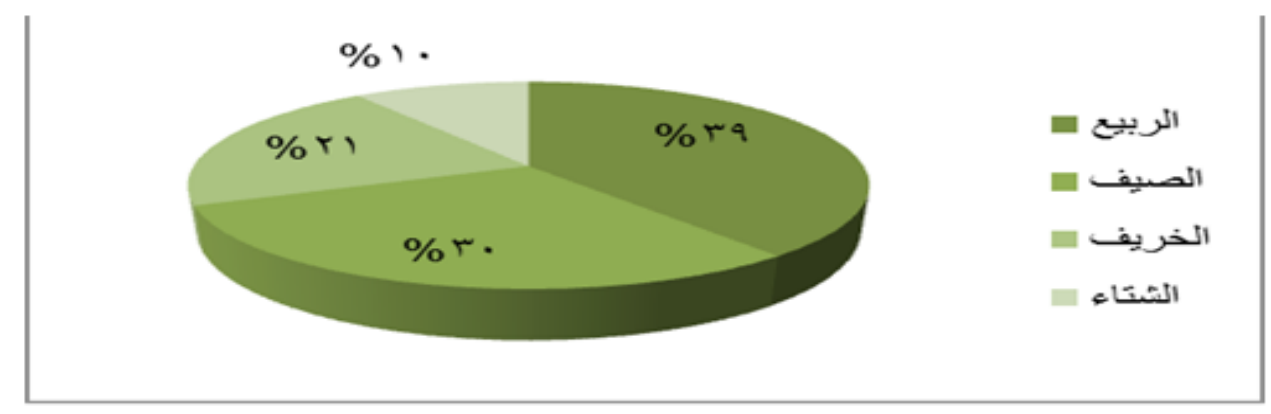

شكل(ع): بيبِن النسبة المئويةَ الموسميَّة للافقريات المقاعِية في الاراسة الحالبية. 
توضح الجداول (2, 3, 4, 5) التغايرات الفصلية للعوامل الفيزيائية والكيميائية المدروسة خلال مدة الدراسة. حيث سجلت اعلى قيم

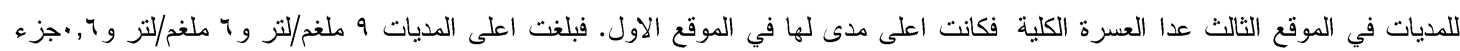

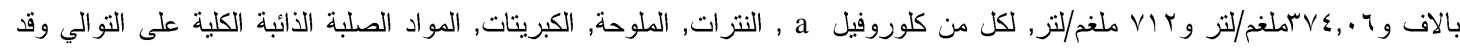
بلغت اعلى قيمة للمتطلب الحيوي للاوكسجين ه.,. •rملغم/لتر في الموقع الثالث ايضا حيث ان هذا الموقع و اقع تحت تاثير محطة معالجة المياه

يوضح الثكل(0) معامل الارتباط بين الصفات المدروسة واللافقزيات القاعية, حيث اظهرت النتائج وجود علاقة موجبة بين كل من كلوروفيل a

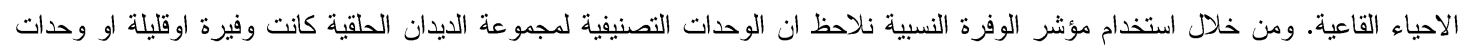
نادرة, فمثلا كانت .Nais sp. gPristina sp وحدات وفيرة في الموقع الثالث وذلك بسبب قدرة هذه الكائنات على تحمل الظروف البيأية القاسية

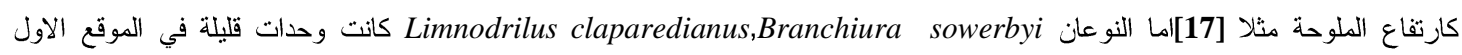

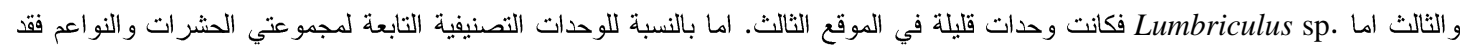
تراوحت مابين وحدات قليلة الى نادرة الى وحدات معدومة, فكانت الوحدات التصنيفية .Dicrotendipes sp, Lymnaea auricularia,

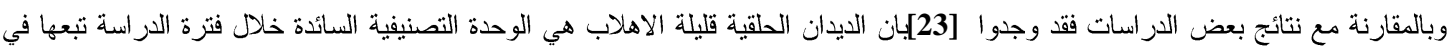
ذلك القتريات و الحشر ات و النو اعم، كما لاحظ [18]فوفرة الديدان الحلقية قليلة الاهلاب وقلة النو اعم وندرة الحشر ات و الديدان الخيطية في شط الحلة،

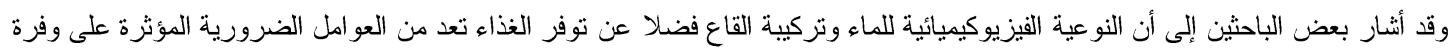
اللافقريات القاعية [25] , [24]. اما بالنسبة لمعامل جاكارد للنتابه بين المواقع المدروسة فكانت ادنى قيمة للتشابه بين الموقع الاول و والثاني V VT, . , اما اعلى قيمة للتشابه بين الموقعين الثاني والثالث وبلغت

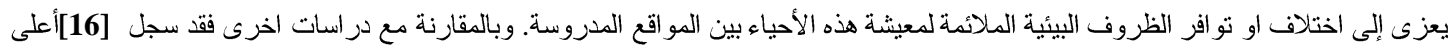

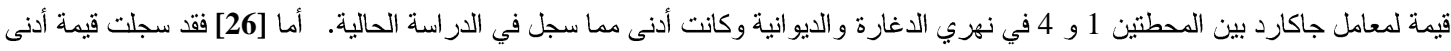

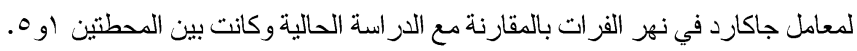

جدول (ץ): يبين التغايرات الفصلية للعو امل المدروسة في الموقع (1).

\begin{tabular}{|c|c|c|c|c|}
\hline خريف & شتثاء & ربيع & صيف & العامل البيئي \\
\hline 17 & 1 & $1 . r$ & $r$ & $\mathrm{BOD} 5(\mathrm{mg} / \mathrm{L})$ \\
\hline$\cdot, Y$ & 1 & 7 & 0 & $\begin{array}{c}\text { Chlorophyll a } \\
(\mathrm{mg} / \mathrm{L})\end{array}$ \\
\hline Y. .7 & $\varepsilon$ & 1 & 1.9 & $\mathrm{NO} 3(\mathrm{mg} / \mathrm{L})$ \\
\hline .00 & . ro & $\because \varepsilon$ & $\because r_{0}$ & Salinity(ppt) \\
\hline rM & rT. & TrI & 1199 & $\mathrm{SO}^{2}=(\mathrm{mg} / \mathrm{L})$ \\
\hline$T \vee \wedge$ & $\leqslant 99$ & $0 \leqslant \leqslant$ & $\leqslant 10.0$ & $\mathrm{TDS}(\mathrm{mg} / \mathrm{l})$ \\
\hline$\sum 77$ & Orร.9人r & rVA & rVA & $\begin{array}{c}\mathrm{TH}(\mathrm{CaCO} 3) \\
(\mathrm{mg} / \mathrm{L})\end{array}$ \\
\hline
\end{tabular}


جدول (ץ): يبين التغايرات الفصلية للعوامل المدروسة في الموقع (Y).

\begin{tabular}{|c|c|c|c|c|}
\hline خريف & 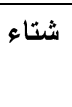 & ربيع & صيف & والعامل البيئي \\
\hline $1 \% . \varepsilon$ & $r .9$ & 1.00 & r & BOD5(mg/L) \\
\hline. .9 & r & v & 7 & $\begin{array}{c}\text { Chlorophyll a } \\
\text { (mg/L) }\end{array}$ \\
\hline$\varepsilon .7$ & Y.o & 1.0 & 1.0 & $\mathrm{NO} 3(\mathrm{mg} / \mathrm{L})$ \\
\hline .7 & o to & $\cdot . \varepsilon$ & מס & Salinity(ppt) \\
\hline ret & rr. & Tro & TYE & $\mathrm{SO}^{2}=(\mathrm{mg} / \mathrm{L})$ \\
\hline 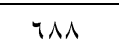 & $\sum 91$ & 041 & $\sum 99$ & TDS(mg/l) \\
\hline$\leq V q . \leqslant \leq Y$ & $\varepsilon r$. & एवs & \&Or & $\begin{array}{c}\mathrm{TH}(\mathrm{CaCO} 3) \\
(\mathrm{mg} / \mathrm{L})\end{array}$ \\
\hline
\end{tabular}

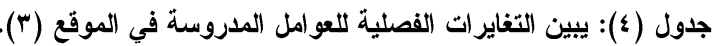

\begin{tabular}{|c|c|c|c|c|}
\hline خريف & 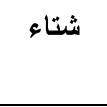 & ربيع & صيف & والعامل البيئي \\
\hline$r . . .0$ & 1.0 & 1.10 & r & BOD5(mg/L) \\
\hline .7 & $\varepsilon$ & 9 & $y$ & $\begin{array}{l}\text { Chlorophyll a } \\
(\mathrm{mg} / \mathrm{L})\end{array}$ \\
\hline 7 & r.r & r & r.r & $\mathrm{NO} 3(\mathrm{mg} / \mathrm{L})$ \\
\hline. .7 & .ro & $\because \leqslant 0$ & .ro & Salinity(ppt) \\
\hline$r V \varepsilon \ldots T$ & $r \backslash \leq$ & $r \leqslant r$ & Trt & $\mathrm{SO}^{2}=(\mathrm{mg} / \mathrm{L})$ \\
\hline VIT & 011 & 00. & or. & $\mathrm{TDS}(\mathrm{mg} / \mathrm{l})$ \\
\hline 01. & EYY.TV & $\varepsilon V \leqslant$ & $\{01$ & $\begin{array}{c}\mathrm{TH}(\mathrm{CaCO} 3) \\
(\mathrm{mg} / \mathrm{L})\end{array}$ \\
\hline
\end{tabular}

جدول(•): يبين مديات العوامل الفيزيائية والكيميائية خلال الدراسة الحالية للمواقع الثلاثة.

\begin{tabular}{|c|c|c|c|}
\hline $\mathbf{S 3}$ & S2 & S1 & العامل البيئي ووحدة قياسه \\
\hline$r . . .0-1.10$ & $1 \% .\{-1.00$ & $17-1$ & BOD5(mg/L) \\
\hline $9-. .7$ & $v-. .9$ & $i-. . Y$ & Chlorophyll a $(\mathrm{mg} / \mathrm{L})$ \\
\hline $1-r$ & $\{.7-1.0$ & $\varepsilon-1$ & $\mathrm{NO} 3(\mathrm{mg} / \mathrm{L})$ \\
\hline . & $.7-. .70$ & $. .00-. .40$ & Salinity(ppt) \\
\hline$r V \varepsilon . . T-r \mid \varepsilon$ & TVT-Yr. & rmו-Irq & $\mathrm{SO} 4=(\mathrm{mg} / \mathrm{L})$ \\
\hline$V \mid r-011$ & $\uparrow \wedge \Lambda-\leqslant ৭ १$ & $T \vee \wedge-\varepsilon \wedge 0.0$ & TDS(mg/l) \\
\hline $01 .-\varepsilon Y 4.7 Y$ & $\leqslant \vee q . \leqslant \leqslant r-r q \varepsilon$ & Or $\$ .9 \wedge r-r V \wedge$ & TH (CaCO3) (mg/L) \\
\hline
\end{tabular}




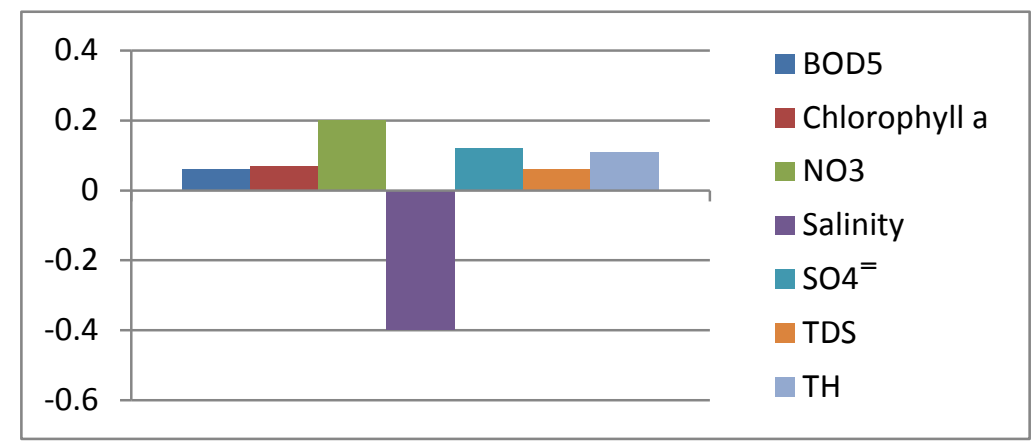

شكل(ه): يبين معامل الارتباط بين الصفات المدروسة و اللافقريات القاعية.

جدول(7): يبين مؤشر الوفرة النسبية للوحدات التصنيفية

\begin{tabular}{|c|c|c|c|}
\hline Taxa & S1 & S2 & S3 \\
\hline \multicolumn{4}{|l|}{ Annelida } \\
\hline Branchiura sowerbyi & $* *$ & * & $* *$ \\
\hline Dero digitata & * & * & $*$ \\
\hline Limnodrilus claparedianus & $* *$ & * & $* *$ \\
\hline Limnodrilus hoffmeisteri & * & * & $*$ \\
\hline Lumbriculus sp. & $*$ & * & $* *$ \\
\hline Nais sp. & * & $*$ & $* * *$ \\
\hline Pristina sp. & $*$ & * & $* * *$ \\
\hline Tubifex tubifex & $*$ & $*$ & $*$ \\
\hline \multicolumn{4}{|l|}{ Insecta } \\
\hline Chironomus(larvae+pupa) & - & $*$ & $*$ \\
\hline Chrysops(larvae) & * & - & - \\
\hline Cryptochironomus sp. & $*$ & $*$ & $*$ \\
\hline Dicrotendipes sp. & * & $* *$ & $*$ \\
\hline Glyptoendipes sp. & $*$ & $*$ & $*$ \\
\hline Microtendipes sp. & * & $*$ & * \\
\hline Polypedilum sp. & $*$ & $*$ & $*$ \\
\hline \multicolumn{4}{|c|}{ استمر ار جدول (7 ) } \\
\hline \multicolumn{4}{|l|}{ Mollusca } \\
\hline Bellamya bengalensis & $*$ & * & $*$ \\
\hline Corbicula fluminalis & $*$ & $*$ & $*$ \\
\hline Corbicula fluminea & - & $* *$ & $*$ \\
\hline Lymnaea auricularia & $* *$ & - & $*$ \\
\hline
\end{tabular}




\begin{tabular}{|c|c|c|c|}
\hline Melanopsis costata & - & $*$ & $*$ \\
\hline Melanopsis nodosa & $*$ & $*$ & - \\
\hline Theodoxus jordani & $*$ & - & $*$ \\
\hline Turritella sp. & $*$ & $*$ & $*$ \\
\hline Unio tigridis & $*$ & $*$ & $*$ \\
\hline Viviparus bengalensis & $*$ & $*$ & $*$ \\
\hline Nematoda & & & $*$ \\
\hline Aphanolaimus sp. & - & $*$ & $*$ \\
\hline Dorylaimus sp. & - & $*$ & $*$ \\
\hline Trilobus longus & - & & $*$ \\
\hline
\end{tabular}

$$
\text { - وحدات غير موجودة,** وحدات نادرة,** وحدات قليلة,*** وحدات وفيرة. }
$$

جدول(V) يبين معامل جاكارد للتشابة

\begin{tabular}{|c|c|c|}
\hline S3 & S2 & , المحطات \\
\hline$\cdot, \mathrm{V} \leqslant$ & $\cdot, \mathrm{V}$ & S1 \\
\hline,, ^॰ & & S2 \\
\hline
\end{tabular}

\section{CONFLICT OF INTERESTS}

There are no conflicts of interest.

[1] F.Hauer and V. Resh, Macroinvertebrates.In: Hauer, F.R.\& Lamberti, G.A. Methods in Stream Ecology, 2006.

[2] J.Olomukoro, "Salinity and the Macrobenthic Community Structure in Eichhornia crassipes of Warri River, Nigeria" . Journal of Biol. Sci., vol. 7, no. 2, pp. 309-314, 2007.

[3] T. Widerholm, "An exotic oligochaete, Branchiura sowerbyi Beddard, in a warm water effluent in Lake Maluren National Swedish Environmental Protection Board, Limnol. Surv. Uppsasala, vol. 37, 1-4, 1970.

[4] P. Kripa, K. Prasanth, K. Sreejesh and T. Thomas. "Aquatic Macroinvertebrates as Bioindicators of Stream Water Quality - A Case Study in Koratty, Kerala, India”. Journal of Research of Recent Sciences, vol. 2, pp. 217222, 2013.

[5] TCEQ (Texas Commission on Environmental Quality), A guide to Freshwater Ecology. Published \& distributed by the: TCEQ, PO Box 13087, Austin, TX 78711-3087, 2005.

[6] M. Ahmed, "Systematic study on mollusca from Arabian Gulf and Shatt Al- Arab, Iraq". Center for Arab Gulf studies,Univ. Basrah., Iraq, 105p. 1975.

[7] M.Al-Saffar, "Interaction between the Environmental Variables and Benthic Macroinvertebrates Community Structure in Abu Zirig Marsh, Southern Iraq". M.S. thesis, Collage of Science, University of Baghdad, Baghdad, Iraq, 2006. 
[8] M.Al-Haidarey, "Duirnal Variation of heavy metals in Al-Kufa River/ Najaf Iraq", 10th conference of biogeochemistry of trace element, Maxico, 2010.

[9] APHA (American puplic Helth Association), Standard methods for examination of water and wastwates, 2 th, E.d. U.S.A., Washington DC, 2003.

[10] R. Smith, Current methods in aquatic science, Canada, University of Waterloo, 2004.

[11] W. Edmondson, Freshwater biology, 2nd Ed. John Wiley and Sons, New York, Freshwater Ecol. 18: 383-393, 1959.

[12] J.Plaziat and W.Younis, "The modern environments of Molluscs in southern Mesopotamia, Iraq: A guide to paleogeographical reconstructions of Quaternary fluvial, palustrine and marine deposits". CG. Notebooks on Geology-A01: 1-18.2005.

[13] M. Dobson, S. Pawley, M. Fletcher, and A. Powell, Guide to Freshwater Invertebrates.Freshwater Biological Association, Scientific Publication, 68,UK. 2012.

[14] K. Nyakeya, R. Okoth, M. Onderi, and G. John, "Assessment of pollution impacts on the ecological integrity of the Kisian and Kisat Rivers in Lake Victoria drainage basin, Kenya", Journal of Afri. of Environ. Sci. and Techno, vol. 3 no.4, pp. 97-107, 2009.

[15] J.Stribling, B. Jessup, J. White, Boward, and D.Hurd, "Development of a Benthic Index of Biotic Integrity for Maryland Streams". Report no. CBWP- EA- 98-3. Annapolis, MD 21401. 1998.

$$
\begin{aligned}
& \text { [16] هاحب, إبر اهيم, "التتوع الإحيائي للافقريات في نهري الدغاره والايو انية، العر اق". أطروحة دكتور راه، كلية التربية، جامعة القادسية, العراق, } \\
& \text {.r... } \\
& \text { [17] اسيل .الربيعي وعلي. اللامي, و مهند. نثات, "التغيرات المكانية و الزمانية لتركيب مجتمع لافقريات القاع في الجزء الثمالي من المصب }
\end{aligned}
$$

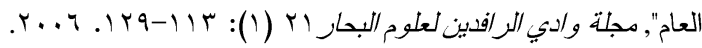

$$
\begin{aligned}
& \text { [18] علي.الفنهر اوي, "توزيع وتتوع اللافقريات القاعية الكبيرة في رواسب شط الحلة/ العراق". رسالة ماجستير , كلية العلوم, جامعة بابل, }
\end{aligned}
$$

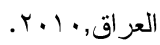

[19] D. Williams, and B. Feltmate, "Aquatic insects". CAB International:358. 1992.

[20] A. Zullini, "Nematodes As Indicators Of River Pollution". Journal of Nematol. Medit. vol. 4, pp. 13-22, 1976.

[21] J. Hyland, C. Cooksey, L. Balthis, M. Fulton, and D. Bearden. "Survey of Benthic Macroinfauna and levels of Chemical Contaminants in Sediments and Biota at Grays Reef NationalMarin Savannh", GA.22PP. 2002.

[22] P. Corbet, "Dragonflies: Behavior and Ecology of Odonata". Harley books, Colchester, 1999.

[23] A. Radhi, A. Al-Lami, and M. Nashaat, "Ecology and diversity of benthic invertebrate at Al-Radwania drainage canal. Basrah”, Journal of Agric. Sci., vol. 17, no.2, pp. 255-264, 2004.

[24] N. Zabbey and A.Hart,"Influence of some physicochemical parameters on the composition and distribution of benthic fauna in Woji Creek Niger Delta, Nigeria”, Journal of Global. Pure Applied Sci. Vol. 12, pp. 1-5, 2006.

[25] K. Koli and D. Muley, "Study of Zooplankton and seasonal variation with special reference to physicchemical parameters in Tulshi reservoir of Kolhapur District (M.S.), India, E-Int". Journal of Sci. Res., vol. 6, no. 1 , pp. $38-46,2012$.

[26] [أمل. شكرخان, "در اسة بيئية لمجتمع اللافقريات القاعية لنهر الفرات في منطقة سدة الهندية".أطروحة دكتور اه، كلية العلوم, الجامعةالمستتصرية، 